\title{
Elementary Logic from an Advanced Standpoint
}

\author{
Maurice A. Finocchiaro \\ Department of Philosophy \\ University of Nevada at Las Vegas \\ Las Vegas, NV 89154-5028 U.S.A. \\ mauricef@nevada.edu
}

\section{Introduction}

For the past 30 years I have taught a course entitled "Logical Theory," and here I should like to briefly relate its origin and history, describe its structure and content, and explain its aim and rationale. The course is at the upper-division level and has for prerequisite any one of three introductory logic courses in (1) reasoning and critical thinking, (2) evidence and inductive reasoning, or (3) formal, deductive, or symbolic logic. Its catalogue description reads: "General study of the nature of argument; how it relates to reasoning, criticism, deduction, logical form, induction, and persuasion. Emphasizes both the systematic development of logical concepts and their application to actual arguments."

This course originated in part as my attempt to teach a course similar ( $m u$ tatis mutandis) to a graduate seminar I had taken in 1967 from Michael Scriven as a graduate student at the University of California-Berkeley, which was my first exposure to informal logic and critical thinking. That memorable course was enti- 
tled "Elementary Reasoning from an Advanced Standpoint" and described as follows in a class handout distributed on January $31^{\text {st }}, 1967$ :

The logical structure of actual arguments is still strikingly obscure, a fact which is concealed behind the internal precision, the intricacy and the intrinsic interest of the various formal systems which have been spawned in the attempt to clarify that logical structure. The classical formal accounts, from the syllogism through extensional sentential calculi to the systems S1-S6, are either notoriously or notably deficient, especially with respect to the (i) encoding procedure, (ii) the identification of assumptions and presuppositions, (iii) the circularity of certain definitions of the connectives in terms of the stroke function (or of numbers in terms of iterated quantifiers), (iv) the analysis of implication, (v) the distinction between deductive and inductive arguments and (vi) that between use and mention, (vii) the "paradox of analysis," (viii) the "Achilles and the tortoise" regress, (ix) the nature of argument by analogy and $(\mathrm{x})$ of induction by simple enumeration, (xi) the analysis of "internal" and "external" probability, (xii) general statements, (xiii) "classical" and "criterial" definitions, (xiv) evaluation, etc.

This seminar will examine some well-known proposed treatments of these difficulties, including suggestions by philosophers such as Lewis, Reichenbach, Carnap, Strawson, Ryle, Toulmin, Anderson and Belnap. To provide a basis for such discussions, the seminar will be substantially concerned with the "workshop" task of analyzing elementary arguments with more than usual care. This analysis will be used not only to evaluate the suggestions mentioned, but also to develop a more accurate account of reasoning and a more effective method of teaching the skills involved in it. This will involve some study of relevant psychological data, of innovative elementary texts, and of the relation between psychology and logic. Prerequisite: the capacity to identify $80 \%$ of the topics and people mentioned or unusually high motivation and reasoning capacity. Texts: Toulmin, Uses of Argument and Strawson, Introduction to Logical Theory.

The origin of my course also lies in my teaching experience and involvement with curriculum design and revision at my university. When I was hired (as a specialist in logic and philosophy of science), I was given the task of redesigning our course offerings in this area. In regard to logic, I wanted to have a course with features such as the following. It was to be advanced, at least in the minimal sense of being a course to be taken after some introductory logic course, which would be its prerequisite. It was to be general in the sense of either including both deductive and inductive reasoning and Iogic, or of transcending this distinction. It was to be oriented toward practice and application. And I did not see this orientation as incompatible with theory, but rather I thought sound theorizing had to be based on some domain or set of materials and data outside itself.

Furthermore, the course was to be required of philosophy majors (but open to nonmajors), and so it would have to cover topics and approaches useful and important from a philosophical point of view; here I had to assume some metaphilosophical conceptions. To make a long story short, let me say simply that I did not want to equate philosophy with technical routines and technicalities (such as those that abound in mathematical logic), nor with being primarily concerned with what "philosophers" say or think and how "they" reason.

At first I used as textbooks the works of Toulmin or Strawson used in Scriven's 
course. I also tried such books as Richard B. Angell's Reasoning and Logic (New York: Appleton, 1964), Robert Fogelin's Understanding Arguments (New York: harcourt Brace Jovanovich, 1978), C.L. Hamblin Fallacies (London: Methuen, 1970), and Wesley Salmon's Logic (Englewood Cliffs, NJ: Prentice-Hall, 1963). When Scriven's Reasoning came out, I found it more appropriate to use it in my introductory course on critical thinking, rather than in "Logical Theory." In any case, whatever textbooks I used, they had to be supplemented with other material in various ways, occasionally discussions of concepts and principles, more frequently concrete and realistic examples of arguments. These examples tended to be different from those examined in introductory courses, which properly focus on everyday arguments dealing with current events and issues and found in newspapers and magazines. For the advanced course the examples were longer and more complex. I toyed with the idea of using arguments for and against the existence of God, as found either is a classic source like Hume's Dialogues Concerning Natural Religion or in a contemporary source such as Scriven's Primary Philosophy (New York: McGraw-Hill, 1966, pp. 87-164); but I never did use them in this course. Instead I soon started using as examples the natural-language arguments for and against the motion of the earth that were intensely discussed during the Copernican Revolution, the best collection being Galileo's Dialogue on the Two Chief World Systems. Parts of my book Galileo and the Art of Reasoning (Dordrecht: Reidel, 1980) reflect that early experience, and accordingly after this book was published I used parts of it along with other textbooks.

Eventually I settled on the way I have been teaching the course in the last several years. Now the course has three approximately equal parts. The first part is relatively "theoretical"; in it we study Salmon's Logic as a particular example of a logical theory which corresponds in broad outline to the most common and traditional approach. The second part is relatively "practical" and covers a spectrum of Galilean arguments about the motion of the earth, using as a textbook my new abridged translation and guide entitled Galileo on the World Systems (1997). Here the aim is partly to understand, analyze, and evaluate such argumentation in any way that seems appropriate; partly to apply as many of Salmon's concepts and principles as feasible; and partly to formulate additional concepts and principle that might constitute elements of an alternative. The third part of the course studies a relatively recent contribution to the theory of argument or logical theory as conceived here, and the work studied changes each time; this part of the course also allows me to become better acquainted with recent work, even if I have not done so in the context of research and scholarship. Here I have used such books as Douglas Walton's Informal Logic (Cambridge, 1989), Frans H. van Eemeren et al.'s Fundamentals of Argumentation Theory (Mahwah, NJ: Lawrence Erlbaum, 1996) next time I will probably use Ralph H. Johnson's Manifest Rationality (Mahwah, NJ: Lawrence Erlbaum, 2000).

To elaborate, I provide four things below: (1) the course syllabus and three lists of study questions on (2) Salmon's Logic, on (3) my Galileo on the World 
Systems, and on (4) van Eemeren et al.'s Fundamentals of Argumentation Theory. The class meets twice a week, for periods of 75 minutes each, and for a semester of fifteen weeks; the thirty class days are usually reduced to twenty-eight on account of holidays. The study questions are meant to tell students what to focus on when they study the reading assignments before class, but they are also the subject matter of the lectures, and they also serve (either verbatim or in slightly modified form) as examination questions. The questions whose numbers have an asterisk are relatively more difficult.

All questions are keyed to the section or chapter number of the book to which they refer; the first numeral in the number of a question denotes the section or chapter number. For example, question (5.5) in the Salmon list is the fifth of a group of five questions dealing with section 5 of Salmon's book (assigned for day no. 2); question (3.6) in the "selections" part of the Galileo list is the sixth of a group of six questions dealing with selection no. 3 in the Galileo book (assigned for day no. 17); question (1.6.2) in the appendix part of the Galileo list is the second of a groups of two questions dealing with Appendix 1.6 (assigned for day no. 19); and question (10.9) in the Eemeren list is the ninth of a group of nine questions dealing with chapter 9 of his book (assigned for day no. 28).

\section{Syllabus}

Textbooks: Wesley C. Salmon, Logic, 3rd edition; Galileo Galilei, Galileo on the World Systems, trans. \& ed. by Maurice A. Finocchiaro; Frans H. van Eeemeren et al., Fundamentals of Argumentation Theory.

Schedule:

Day 1 Preliminaries; Salmon, pp. 1-18 (sections 1-4)

2 Salmon, repeat pp. 1-18, and study pp. 19-23 (sect. 5)

3 Salmon, pp. 23-37 (sects. 6-9)

4 Salmon, pp. 37-44 (sect. 10)

5 Salmon, repeat pp. 37-44, and study pp. 44-50 (sects. 11-12)

6 Salmon, pp. 50-63 (sects. 13-14)

7 Salmon, pp. 63-73 (sect. 15)

8 Salmon, pp. 85-97 (sects. 18-23)

9 Salmon, pp. $97-108$ (sects. 24-26)

10 Salmon, pp. 108-18 (sects. 27-28)

11 Salmon, pp. 118-39 (sects. 29-30)

12 Salmon, repeat pp. 127-39 (sect. 30); review everything

13 TEST \# 1

14 Galileo, Introduction, pp. 1-28

15 Galileo, Introduction, pp. 28-69

16 Galileo, Appendix 1.1-1.3 (pp. 309-18) 
17 Galileo, selection 3 (pp. 91-107) and Appendix 1.4 (pp. 318-23)

18 Galileo, sel.5 (pp. 117-28); Intro. 5.4 (pp. 58-62); \& Appendix 2.3 (p. 341-44)

19 Galileo, selection 6 (pp. 128-42) and Appendix 1.6 (pp. 326-30)

20 Galileo, selection 8 (pp. 155-70) and Appendix 1.5 (pp. 323-25)

21 Galileo, selection 11 (pp. 221-44)

22 Galileo, selection 15 (pp. 282-303); review

23 TEST \# 2

24 Eemeren, chapter 1 (pp. 1-26)

25 Eemeren, chapter 6 (pp. 163-88)

26 Eemeren, chapter 7 (pp. 189-212)

27 Eemeren, chapter 8 (pp. 213-45)

28 Eemeren, chapter 10 (pp. 274-311)

29 COMPREHENSIVE FINAL EXAMINATION

\section{Study Questions about Salmon's Logic}

(1.1) What is meant by argument, premise, conclusion, and logical correctness?

(2.1) What is the difference between an inference and an argument?

(3.1) Why is it that showing that a justification is inadequate does not show that the conclusion is false? (3.2) What is the distinction between the context of discovery and the context of justification?

(4.1) What is meant by (a) correct deductive argument, (b) correct inductive argument, (c) deductive argument, (d) inductive argument, and (e) fallacious argument? (4.2) What might be meant by (a) deductively correct argument, and (b) inductively correct argument? (*4.3) On p. 18 Salmon states that "any inductive argument can be transformed into a deductive argument by the addition of one or more premises"; in what sense is this true? Why is it true? Can you give an example (of an inductive argument, the premise(s) to be added, and the deductive argument into which it is transformed)? You might want to try to construct such example by using the proposition "the sun will rise tomorrow" as conclusion.

(5.1) What is meant by valid argument and by invalid argument? (5.2) What are the various truth-value combinations of premises and conclusion that are allowed and that are excluded for (a) valid and for (b) invalid arguments? (5.3) What is the one truth-value combination of premises and conclusion which is excluded in a valid argument? Why? (5.4) What is an "argument form"?

(5.5) What is the method of counterexample? Why does it work?

(6/7.1) What is meant by each of the following: conditional, antecedent, consequent, biconditional, conversion, contraposition, affirming the antecedent, affirming the consequent, denying the antecedent, and denying the consequent? (6/ 
7.2) Which of the argument forms mentioned in (6/7.1) are valid and which invalid? Why? $\left({ }^{*} 6 / 7.3\right)$ On p. 24 Salmon states that "unless' means the same as 'if not ...'; explain what this means; how would you justify Salmon's assertion? How would you criticize it? If 'unless' does not mean what he says, what does it mean?

(8.1) What is meant by "reductio ad absurdum"? How does it differ from denying the consequent? (*8.2) Salmon says that reductio ad absurdum is sometimes valid and sometimes invalid; is this really correct? How can this be? (8.3) Analyze [c] and [f] in terms of schema [a], in the way that Salmon does for [d] and [e]. If such an analysis is impossible, indicate why.

(9.1) What is meant by "dilemma"? What are its special cases? Why is it valid?

(10.1) What does it mean to say that a connective is "truth functional"? (10.2) What is the meaning of the symbols: $\sim . \vee \supset=.(10.3)$ What are the "paradoxes of material implication"? (10.4) How does one test an argument form for validity or invalidity by means of a truth table? (10.5) What is meant by disjunctive syllogism and by hypothetical syllogism? (*10.6) Define the exclusive disjunction first by means of a truth table, and then in terms of the standard symbols (that is, those at the bottom of p. 38); in other words, suppose we let $x$ symbolize the exclusive disjunction; then give the truth-conditions for $\mathrm{p} \times \mathrm{q}$, and show to what combination of standard symbols $\mathrm{p} x \mathrm{q}$ corresponds. $\left({ }^{*} 10.7\right)$ Give a truth table definition of neither ... nor; that is, give the truth conditions for neither $\mathrm{p}$ nor q; also define neither ... nor in terms of the standard symbols. (10.8) Show by truth tables that the three versions of reductio ad absurdum (as redefined) are valid. (10.9) Show that the connective "because" is not truth-functional by giving an example of a true because-statement whose two parts are true, and an example of a false because-statement whose two parts are also true. (10.10) Discuss the connection between the symbol " $\supset$ " (material conditional) and the connective "ifthen" by seeing whether you can give an argument similar to that of (10.9) to show that "if-then" is not truth-functional. (10.11) Check by truth tables the validity of all argument forms named in the book or in class in regard to sections 6-10.

(11.1) How does one use a truth table to show the logical equivalence of two complex statement forms? (11.2) How does one use a logical equivalence between two complex statement forms, to show that the validity of a given argument form reduces to the validity of a previously decided argument form? (11.3) What does it mean to speak of sufficient and of necessary conditions? (11.4) Do you agree with Salmon (pp. 46-47) that "unless" means "or"?

(12.1) What is a tautology? (12.2) How does one use a truth table to determine whether a given statement form is a tautology? (12.3) What is an important connection between logical equivalences and tautologies? (12.4) What is an important connection between the validity of an argument form and the notion of a tautology? 
(13.1) What is meant by the following types of statements: categorical, universal affirmative, universal negative, particular affirmative, and particular negative? What is meant by a subject term and by a predicate term? (13.2) What is the meaning attached to each type of categorical statement? (13.3) What are the logical connections among the four types of categorical statement with the same subject and same predicate: all $\mathrm{S}$ are $\mathrm{P}$, no $\mathrm{S}$ are $\mathrm{P}$, some $\mathrm{S}$ are $\mathrm{P}$, and some $\mathrm{S}$ are not $P$ ? (13.4) What are the various ways of expressing in common language each of the categorical statements?

(14.1) What is meant by: categorical syllogism, end term, and middle term? (14.2) What is the fallacy of division and the fallacy of composition? (14.3) What is meant by saying that a term is "distributed"? (14.4) Why is it that (a) subject terms of universal statements and (b) predicate terms of negative statements are distributed? (14.5) What are the three rules for testing syllogisms? (*14.6) Why do these rules work? Why are they right? (14.7) What is a quasi-syllogism? How does it differ from a syllogism? Why is it valid? (14.8) Check for validity by using the method of distribution rules: (a) all $\mathrm{A}$ are $\mathrm{B}$, all $\mathrm{B}$ are $\mathrm{C}$, so all $\mathrm{A}$ are $\mathrm{C}$; (b) no $\mathrm{A}$ are $\mathrm{B}$, no $\mathrm{B}$ are $\mathrm{C}$, so no $\mathrm{A}$ are $\mathrm{C}$; (c) some $\mathrm{A}$ are $\mathrm{B}$, some $\mathrm{B}$ are $\mathrm{C}$, so some $A$ are $C$; and (d) some A are not B, some B are not C, so some A are not C. (14.9) Discuss whether and why the following syllogism is valid or invalid: all metals are solid; all solids are rigid; therefore, some metals are rigid.

(15.1) What is the Venn diagram for each of the four types of categorical statements? (15.2) How do you construct the Venn diagram for a syllogism? (15.3) How do you use Venn diagrams to check whether a given syllogism is valid or invalid? (15.4) Check for validity by using the method of Venn diagrams: (a) all $\mathrm{A}$ are $\mathrm{B}$, all $\mathrm{B}$ are $\mathrm{C}$, so all $\mathrm{A}$ are $\mathrm{C}$; (b) no $\mathrm{A}$ are $\mathrm{B}$, no $\mathrm{B}$ are $\mathrm{C}$, so no $\mathrm{A}$ are $\mathrm{C}$; (c) some $\mathrm{A}$ are $\mathrm{B}$, some $\mathrm{B}$ are $\mathrm{C}$, so some $\mathrm{A}$ are $\mathrm{C}$; and (d) some $\mathrm{A}$ are not $\mathrm{B}$, some $\mathrm{B}$ are not $\mathrm{C}$, so some $\mathrm{A}$ are not $\mathrm{C}$.

(19.1) What are two differences between deductive correctness and inductive correctness?

(20.1) What is the argument form called "induction by enumeration"?

(21.1) Under what conditions does an induction by enumeration become a fallacy of insufficient statistics?

(22.1) Under what conditions does an induction by enumeration become a fallacy of biased statistics?

(*20-22.1) Construct three inductions by enumerations based on true premises and with the following conclusions: (a) "p unless q" means "p iff not-q"; (b) "all A are B" means "for each $x$, ( $x$ is $A \supset x$ is B)"; and (c) "if $p$ then q" means " $p \supset q$ "; then discuss the inductive correctness of these arguments and especially whether they commit the fallacies of insufficient and of biased statistics.

(23.1) Define the argument form called "statistical syllogism"? (23.2) Under what conditions does a statistical syllogism become a fallacy of incomplete evidence? 
(24.1) Let [a] rather than [b] be the definition of "argument from authority." What are several conditions under which an argument from authority is fallacious? (24.2) What is the argument form called "argument from consensus"? Here make a change similar to that suggested in (24.1).

(25.1) Change Salmon's definition of "argument against the person" by deleting the first line of [a] on p. 102; under what conditions then are arguments against the person inductively correct? (25.2) What is the argument form called "negative argument from consensus"?

(26.1) What is the argument form called "argument by analogy"? (26.2) What conditions determine the correctness or incorrectness of arguments by analogy?

(27.1) What is meant by a causal argument?

(28.1) What is meant by saying that a causal argument is using: the method of agreement, the method of difference, the joint method of agreement and difference, and the method of concomitant variation? (28.2) For each special type of causal argument, what determines whether it is inductively correct?

(29.1) Under what conditions does a causal argument commit: the fallacy of post hoc, the fallacy of confusing cause and effect, and the fallacy of the common cause.

(30.1) What is meant by: hypothesis, confirmatory instance, and disconfirmatory instance? (30.2) What is the "hypothetico-deductive method"? (30.3) What are the "statements of initial conditions"? (30.4) What are "auxiliary hypotheses"? $(* 30.5)$ Structure [a] on p. 128 as a combination of arguments having previously studied forms, such as quasi-syllogisms and conditional arguments; do something analogous for [b] on p. 129, one obviously relevant argument form being the statistical syllogism. $\left({ }^{*} 30.6\right)$ What previously studied argument form turns out to be very common in science, even though it is deductively incorrect? What are two conditions that need to be satisfied if such a common scientific argument is to be logically correct? Do these conditions make it deductively correct or only inductively correct?

\section{Study Questions about Galileo on the World Systems}

\section{A. Selections}

(3.1) (a) What is the main Aristotelian argument being criticized in this passage? (b) How does this Aristotelian argument support the conclusion that the heavens are unchangeable? (c) In what sense in this argument anti-Copernican? (3.2) (a) What flaws does Galileo attribute to this Aristotelian argument? (b) For each flaw, how does Galileo try to show that the Aristotelian argument is flawed in that manner? (3.3) How plausible is each Galilean criticism of the Aristotelian argument? (3.4) Do the various Galilean criticisms have something in common, and if so what is it? If not, why not? (3.5) (a) Besides criticizing this Aristotelian argument, is Galileo also advancing a conclusion about the physical world? If so, 
(b) what is this conclusion, and (c) how does he support this conclusion? (3.6) (a) Besides criticizing this Aristotelian argument, is Galileo also advancing a general conclusion about human knowledge? If so, (b) what is this conclusion, and (c) how does he support or illustrate this conclusion?

(5.1) (a) How does Galileo criticize the Aristotelians? (b) What faults does he attribute to the Aristotelians? (c) What examples does Galileo give to illustrate these faults? (*5.2) (a) Do all these faults have something in common, and if so what is it? (b) How does this criticism of the Aristotelians relate to the Copernican controversy? (5.3) (a) What general conclusion about human knowledge is Galileo advancing? (b) How is he supporting or illustrating this conclusion?

(6.1) One of the things Galileo does here is to advance a general conclusion about human knowledge, which may be called the principle of simplicity; (a) how would you state this Galilean principle of simplicity? (b) does Galileo give any reasons why we should accept this principle? (6.2) (a) Explain some of the reasons why Galileo claims that it is simpler to attribute the diurnal rotation to the earth rather than to the rest of the universe; (b) can you think of any way of criticizing this Galilean reasoning, perhaps along the lines mentioned by Simplicio on pp. [148-49]? (*6.3) The third argument presented by Galileo (pp. [144-45]) is interesting and important in its own right, independently of its connection with the rest of the passage; explain how this argument tries to show that it is more probable for the earth than for the rest of the universe to have the diurnal rotation. (6.4) One of the things Galileo does here is to advance a conclusion about the physical world, which may be called the principle of the relativity of motion; (a) how would you state this Galilean principle? (b) how does he justify or illustrate this principle?

(8.1) In this selection Galileo states and criticizes three distinct (though related) arguments against the earth's motion, and all three involve different aspects of vertical fall; (a) state the argument from actual vertical fall; (b) summarize and explain Galileo's criticism of the argument from actual vertical fall; (c) state the argument from apparent vertical fall; (d) summarize and explain Galileo's criticism of the argument from apparent vertical fall; (e) state the ship analogy argument; (f) summarize and explain Galileo's criticism of the ship analogy argument. (8.2) In this selection Galileo states and uses two important physical principles; (a) state the principle of the conservation of motion; (b) how does Galileo use this principle? (c) how does he justify it? (d) state the principle of the superposition of motion; (e) how does he use this principle? (f) how does he justify it? (8.3) Is Galileo advancing a methodological principle about the relationship between experimental observation and intellectual theorizing? If so, state the principle. How does he support or illustrate this principle? $\left({ }^{*} 8.4\right)$ Galileo seems to use either Plato's doctrine of recollection or the Socratic method? How and where does this happen? What does Galileo believe about it?

(11.1) What conclusion is Galileo advancing about the problem of how best to define what it means to talk about the "center of the universe"? (11.2) (a) What conclusion is Galileo advancing about which body (sun or earth) is located at the 
center of the orbital revolutions of the planets? (b) Which bodies are being counted as planets in this context? (c) How does he justify this conclusion? (11.3) (a) What further conclusion does Galileo advance, in regard to whether the annual motion belongs to the sun or the earth (namely, whether the sun moves in an orbital revolution around the earth once a year, or vice versa); (b) how does Galileo use his earlier conclusion about which body is located at the center to support his further conclusion about whether the annual motion belongs to the sun or to the earth? (11.4) One traditional objection to the earth's annual motion was based on the apparent size of the planet Mars; (a) how did this objection try to disprove the earth's annual motion? (b) how did Galileo answer this objection? (11.5) Another traditional objection to the earth's annual motion was based on the appearance of the planet Venus; (a) how did this objection try to disprove the earth's annual motion? (b) how did Galileo answer this objection? (11.6) A third traditional objection to the earth's annual motion was based on the orbit of the moon; (a) how did this objection try to disprove the earth's annual motion? (b) how did Galileo answer this objection? (11.7) (a) What general conclusion does Galileo advance about the relationship between sense experience and reason? (b) How does he justify or illustrate this conclusion?

(15.1) (a) How do acceleration and retardation cause water to move with tidallike motions? (b) How does the combination of the earth's two motions cause a daily acceleration and retardation for each point on the earth, which gets the process of ocean tides started? (c) What are some of the fluid properties of water, which act as secondary causes of the tides? (d) What are some additional particular tidal phenomena, and how are they each explained? (15.2) (a) Analyze the basic structure of Galileo's tidal argument in support of the earth's motion by discussing the sense in which the argument may be interpreted as being a causal, explanatory, and hypothetico-deductive argument; (b) analyze the intent of Galileo's argument by discussing whether or not he claims it to be conclusive (i.e., deductive or inductive), and if not, what degree of strength he claims it to have. (*15.3) Evaluate Galileo's tidal argument by presenting and discussing some objections which can be raised against it; for example, one could object that today we know that the tides are caused by the moon's gravitational attraction, and so Galileo's geokinetic explanation of the tides is incorrect; how does this affect the logical correctness of Galileo's reasoning?

\section{B. Appendix}

(1.1.1) What is the meaning of each of the following concepts, and how do they interrelate to one another: reasoning; reasoning indicators; arguments; propositions; conclusions, premises, reasons; arguing that, for, against, and from; objections; counterarguments; and perspective in argumentation.

(1.2.1) What is the meaning of each of the following concepts, and how do they interrelate to one another: serial structure; intermediate and final propositions; 
propositional structure and structure diagrams; standard labeling; linked and independent reasons; rules for structure diagrams and standard labeling; latent propositions; and reconstructions. (1.2.2) Using standard labeling, construct structure diagrams for the following arguments in the following thirteen paragraphs of selection \#7: (1) p. 142, last paragraph, to p. 143, first paragraph; (2) p. 143, second paragraph; (3) p. 143, 3rd paragraph; (4) p. 143, 4th paragraph, continuing to top of p. 144; (5) p. 144, second paragraph; (6) p. 145, 3rd paragraph; (7) p. 145, 4th paragraph to top of page 146 ; (8) p. 146, 2nd paragraph; (9) p. 146, 3rd paragraph; (10) p. 146, 4th paragraph continuing to top of p. 147 ; (11) p. 153 , 3rd paragraph; (12) p. 153, 4th paragraph; and (13) p. 154, 1st paragraph.

(1.3.1) What is the meaning of each of the following concepts, and how do they interrelate to one another: evaluation as bipolar, continuous, and ratiocinative; primacy of negative evaluation; conclusion refuting criticism; premise refuting criticism; premise undermining criticism; reason relevance criticism; disconnection; formal disconnection or deductive invalidity; explanatory disconnection; presuppositional disconnection; internal criticism; semantical disconnection; and persuasive disconnection.

(1.4.1) Apply the technical framework of sections 1.1, 1.2, and 1.3 of the Appendix to the observational argument for heavenly unchangeability. (1.4.2) Consider the observational argument for heavenly unchangeability: (a) give a reconstruction of this argument; (b) analyze this argument by means of a structure diagram, explaining what its propositional structure is, and discussing its latent as well as its explicit structure; (c) evaluate this argument by reconstructing Galileo's criticisms in terms of the types of criticism discussed in Appendix 1.3.

(1.5.1) Apply the technical framework of sections 1.1, 1.2, and 1.3 of the Appendix to the anti-Copernican argument from vertical fall. (1.5.2) Consider the anti-Copernican argument from vertical fall: (a) give a reconstruction of this argument; (b) analyze this argument by means of a structure diagram, explaining what its propositional structure is, and discussing its latent as well as its explicit structure; (c) evaluate this argument by reconstructing Galileo's criticisms in terms of the types of criticism discussed in Appendix 1.3.

(1.6.1) Discuss what is meant in general by "self-reflective" reasoning and argumentation, and give an illustration of a self-reflective argument. (1.6.2) Consider Galileo's simplicity argument for terrestrial rotation: (a) give a reconstruction of this argument; (b) analyze this argument by means of a structure diagram, explaining what its propositional structure is, and discussing its latent as well as its explicit structure; (c) evaluate this argument by developing various points made in Galileo's self-reflections and by applying as many as possible of the various types of criticism discussed in Appendix 1.3.

(*1.7.1) Apply the various methods of analysis and of criticism discussed in Appendix 1.1,1.2, and 1.3 to the various arguments and counterarguments reconstructed in this section (1.7) of the Appendix. 
(1.8.1) Discuss the meaning and interrelations of the following concepts: thinking, reasoning, critical reasoning, critical thinking, analysis, evaluation, and self-reflection.

\section{Study Questions about Fundamentals of Argumentation Theory}

(1.1) Discuss the concepts of serial structure, linked reasons, and independent reasons by explaining the meaning of these terms and giving examples of each (pp. 16-19; see Galileo, pp. 311-13). (1.2) Discuss the aim of and relationships among logical theory, argumentation theory, and formal logic, especially in terms of the distinctions between the descriptive and the normative and between the empirical and the conceptual.

(6.1) Discuss the main concerns and problems of informal logic and how it relates to logical theory and argumentation theory. (6.2) Name the main scholars who have contributed to informal logic and briefly describe their contributions (book authored and idea advanced). (6.3) Discuss what critical thinking is and how it relates to informal logic and to logical theory by stating, comparing and contrasting, and evaluating several main views; besides discussing the views in van Eemeren, chapter 6, discuss Finocchiaro's conception of critical thinking and of its relationship to logical theory, found in Galileo, pp. 308-18, 326-27, 334-35, and 335-39. (6.4) Name the main scholars who have contributed to critical thinking and briefly describe their contributions. (*6.5) Discuss the distinction between the macrostructure and the microstructure (e.g., p. 176) of arguments by explaining what these terms mean and how their distinction and relationship might be used to interrelate formal and informal logic.

(7.1) Discuss the distinction between "conviction" and "persuasion" by explaining their meaning and differences according to some authors, and by evaluating whether the distinction is really sound (pp. 189-90). (7.2) Discuss Zarefsky's definition of argumentation by explaining what it says and how it represents a move toward rhetoric (pp. 191-93). (7.3) Discuss D. O'Keefe's distinction between two senses of "argument" by explaining the meaning of each sense, the relationship between them, and the implications of the distinction for argumentation theory (pp. 197-98). (7.4) Discuss the problem of "fields of argument" by explaining what the issues are and by summarizing Goodnight's views on "spheres of argument" and on the interaction among personal, technical, and public spheres (pp. 204-7).

(8.1) Compare and contrast Aristotle's definition of "syllogism" (pp. 214, 223) with Salmon's definition of "syllogism" and with the definition of deductive correctness. (Hint: Aristotle's definition of syllogism may be regarded as a definition of a special kind of "logical correctness" which may be called "syllogistic correctness"; then the definition would read that an argument is "syllogistically correct" if and only if the several Aristotelian conditions apply. One issue is that one of the 
Aristotelian conditions is equivalent to deductive correctness. Another issue is the significance of the other Aristotelian conditions. A third issue is that Salmon's definition of "syllogism" does not even include the first Aristotelian condition.) $\left({ }^{*} 8.2\right)$ On pp. 228-32 van Eemeren et al. claim that although there is no equivalence between natural-language statements and truth-functional expressions of symbolic propositional logic, there is some correspondence; that is, while it is not the case that whenever a truth-functional expression is true the corresponding natural-language statement is also true, it is the case that whenever a truth-functional expression is false the corresponding natural-language statement is also false; then on the basis of this claim they seem to argue that this correspondence is enough to ensure that if a truth-functional symbolized argument is formally valid the corresponding argument in natural language is also formally valid. Explain (or reconstruct) the details of their argument and evaluate its correctness. $(* 8.3)$ On pp. 232-34, van Eemeren et al. discuss the fact that there are arguments "which are valid even though their logical form is invalid" (p. 232), and on the basis of this fact they argue that this implies the paradox that "whether an argument is formally valid is not itself an entirely formal matter" (p. 234); explain (and/or reconstruct) the details of their argument and evaluate its correctness. (Hint: Compare and contrast this argument to the discussion in Salmon, class, and written homework on the distinction between deductive correctness and formal validity, on the fact that there are deductively correct arguments which are not formally valid, and on the claim that formally invalid but deductively correct arguments can be transformed into formally valid arguments by adding to their premises.) (8.4) Discuss the Woods-Walton concepts of "formal fallacy" (p. 220), "formally explicable fallacy" (p. 222), and "formally analyzable fallacy" (p. 237) by stating, explaining, illustrating, and comparing and contrasting their definitions; discuss the three types of "formalisms" (and corresponding "informalisms") which these concepts generate. (8.5) Discuss Scriven's criticism of formalistic methods in logical theory by explaining (or reconstructing) his objection, evaluating its correctness, and discussing possible ways of answering it (pp. 244-45).

(10.1) Discuss in what sense the pragma-dialectical theory of argumentation is "pragmatic" and in what sense it is "dialectical." (10.2) Discuss van Eemeren and Grootendorst's pragma-dialectical concept of argumentation by stating their definition (p. 275) and by explaining how its alleged essential aim (resolution of differences of opinion) leads them to formulate their ten rules for critical discussion (pp. 283-84) and to their theory of fallacy (pp. 299-303). (10.3) Evaluate van Eemeren and Grootendorst's pragma-dialectical concept of argumentation (p. 275) by examining whether argumentation has other key aims besides, or instead of, the one they allege (resolution of differences of opinion); for example, such aims as the attainment of truth, the attainment of reasonable (and/or intelligent, and/or rational) beliefs, or the understanding of differences of opinion. (10.4) Discuss the methodological approach of the pragma-dialectical theory of argumentation (pp. 275-76) by explaining how its research program includes a descriptive, a norma- 
tive, as well as "a philosophical, a theoretical, an empirical, an analytical, and a practical component" (p. 276); explain what these terms mean and compare and contrast this approach with that of logical theory defined and presented in class. (10.5) Discuss the pragma-dialectical distinction between "resolving a difference of opinion" and "settling a dispute" (pp. 280-81); explain and evaluate this distinction, and compare and contrast it to the distinction (made in some quarters) between persuasion and conviction. (10.6) Compare and contrast the pragma-dialectical notion of "analytical overview" (pp. 288-91) with the notion of "analysis" of an argument presented in class (e.g., Galileo, pp. 311-14). (10.7) Compare and contrast the pragma-dialectical notion of reconstruction (pp. 291-93) with the notion of reconstruction presented in class (e.g., Galileo pp. 314, 323). (10.8) Discuss the pragma-dialectical concept of "fallacy" by stating and explaining its definition (p. 299) and illustrating it with one or more particular cases (for example, "begging the question," p. 301). (*10.9) Compare and contrast the pragmadialectical account of "begging the question" (as violation of its Rule 6, p. 301) and Finocchiaro's account (as a special type of "presuppositional disconnection" called "persuasive disconnection," in Galileo, p. 318). 